Originally published as:

\title{
Mankertz, A.
}

Molecular interactions of porcine circoviruses type 1 and type 2 with its host (2012) Virus Research, 164 (1-2), pp. 54-60.

DOI: 10.1016/j.virusres.2011.11.001

This is an author manuscript.

The definitive version is available at: http://www.sciencedirect.com/ 
1 Molecular interactions of porcine circoviruses type 1 and type 2 with its host

2

3 Annette Mankertz

4

5 Division of Viral Infections, Robert Koch-Institut, Nordufer 20, D-13353 Berlin, Germany 6

7 e-mail: mankertza@rki.de

8 Fon: $+49(0) 3018754-2516$

9 Fax: +49 (0)30 18754-2598/2328

10

11 


\section{Abstract:}

2 This review discusses the molecular interaction of proteins encoded by porcine circoviruses

3 type 1 (PCV1) and type 2 (PCV2) with respect to the putative role for disease induction.

4 Besides protein:protein interactions also proteins binding to PCV DNA are covered.

5 Moreover, new approaches are considered which have been generated by genomic and

6 proteomic techniques. PCV are still an enigma, when communication with the host and

7 induction of disease are concerned. This is remarkably, since comparison of two viruses with

8 a size of less than 2,000 nts should easily identify the molecular trigger responsible for

9 pathogenicity of PCV2. Since scientific life isn't all beer and skittles we have to accept that

10 this conundrum may be solved only in a long time range. The good news is that some

11 common themes become visible and that certain aspects of the cell life cycle have been

12 identified to be involved in interaction with the pathogen. 


\section{Introduction:}

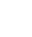

Porcine circovirus type 1 (PCV1) was described in 1974 as a non-pathogenic contaminant of a porcine kidney cell line (Tischer et al., 1986), while the second variant, PCV2, was isolated from diseased pigs 15 years later (Allan et al., 1998; Mankertz et al., 2000; Meehan et al., 1997). PCV2 was identified as the etiological agent of postweaning multisystemic wasting disease (PMWS), a multifactorial disease in swine (Ellis et al., 1998). Both types of porcine circovirus, PCV1 and PCV2, have small viral non-enveloped icosaedric capsids with a diameter of less than $20 \mathrm{~nm}$. Their miniscule ssDNA genomes consist of less than 2,000 nts and express only a few proteins, the gene products of ORF1 (the replicases Rep and Rep', (Mankertz et al., 1998), ORF2 (structural protein Cap (Nawagitgul et al., 2000)) and ORF3 (Liu et al., 2005). Although the pathogenicity of PCV1 and PCV2 could not differ more clearly, both viruses share a high degree of homology with respect to the genomic information. Taking this into account, factors responsible for the pathogenic potential of PCV2 were thought to be easily identified, but more than 15 years of PCV research have not yet provided us with sufficient information to identify a trigger molecule inducing disease. Distinct channels of interaction can be discerned when the relationship of a virus and its host is considered. Infection of a cell will trigger an adaptive reaction of the infectious agent as well as of the host cell. Moreover, the infectious agent must employ strategies to evade the immune answer of the host. This review aims to summarize what has been learned about the interaction of PCV with the host in the last few years.

\section{Host-virus interaction by interaction of viral and cellular proteins}

PCV have a very small genome size and accordingly a highly limited coding capacity. They express only four proteins (Finsterbusch and Mankertz, 2009): Rep and Rep' are transcribed from ORF1 or the rep-gene by alternative splicing. These two proteins are the main factors for initiation of the viral DNA replication (Steinfeldt et al., 2006), although the synthesis 
1 process itself is performed by host proteins. ORF2 encodes the major structure protein Cap,

2 which builds the capsid of the virus. Interestingly, PCV belong to the most stable viruses and disinfection in swine production as well as products from swine is an issue. This became apparent when a vaccine against rotavirus was found to be contaminated with PCV1, although it was demonstrated that the virus was non-infectious (Baylis et al., 2011). The last and smallest viral protein encoded by ORF3 (in the following termed gene product of ORF3, gpORF3) has been described to be involved in modulation of virulence (Chaiyakul et al., 2010).

PCV encoded proteins is one of the interfaces through which the virus interacts with the cell, subverts cellular factors or processes to complete the viral replication cycles and modulates the host activity to clear the virus infection. During the last years, several proteins expressed in porcine cells were identified to interact with Rep/Rep', Cap and gpORF3, mostly using a two-hybrid assay (Finsterbusch et al., 2009; Liu et al., 2007; Timmusk et al., 2006). Description of these interaction partners in the literature is either rather scant when the function of the interacting proteins have not yet been elucidated or overabundant, when proteins with multiple functions have been identified. In consequence, a convincing common ontology of PCV interacting cellular proteins has not yet been recognized, although cellular proteins interacting with Rep, Rep', Cap and gpORF3 are involved in many aspects of viral replication, as e.g. intracellular transport processes and regulation of cell cycle.

Interaction partners of Rep and Rep':

Timmusk et al. have identified two cellular Rep-interacting porcine proteins, a protein similar to human syncoilin. Syncoilin is an intermediate filament (IF) protein. It is highly expressed in skeletal and cardiac muscle (Clarke et al., 2010) and also in the peripheral and central nervous systems. A link between syncoilin and amyotrophic lateral sclerosis has been identified (Wakayama et al., 2010). In addition, interaction of Rep with the transcriptional regulator protein c-myc has been described (Timmusk et al., 2006). This protein is notorious 
1 for its role in Burkitt's lymphoma, which is induced by a cytogenetic abnormality deregulating

2 the myc oncogene. The constitutively active myc drives cell growth and proliferation in

3 Burkitt's lymphoma (Allday, 2009).

4 Three other porcine proteins homologous to human proteins were identified to bind to Rep 5 more recently (Finsterbusch et al., 2009). ZNF265 is an arginine/serine rich domain 6 containing zinc finger protein. It binds to mRNAs and can replace the essential spliceosome 7 factor SF2/ASF (Adams et al., 2001) thereby inducing alternate splicing. It has been shown 8 that other zinc finger proteins such as ZNF569 play a significant role in the immunoregulatory 9 function of animals (Huang et al., 2006). Moreover, ZNF265 expression responds to infection of the testis with the bacterium Ureaplasma urealyticum (Li et al., 2009). The Rep protein resides in the nucleus (Finsterbusch et al., 2005). Co-expression in HEK293 cells led to 12 accumulation of ZNF265 and Rep in point-shaped structures in the nucleus.

13 VG5Q or AGGF encodes an angiogenic factor. Its deregulation is associated with Klippel14 Trenaunay syndrome (KTS), a congenital multisystem disorder characterized by varicose veins, cutaneous capillary malformation, and hypertrophy of bone and soft tissue (Oduber et al., 2008). The expression of $V G 5 Q$ is enhanced by the transcription factor GATA1. Knockdown of GATA1 expression reduced expression of AGGF1, resulting in apoptosis of endothelial cell, inhibition of endothelial capillary vessel formation and cell migration (Fan et al., 2009). Co-expression of Rep and VG5Q showed both proteins in their initial compartment, but the fluorescence signal was reshaped to a more granulous appearance.

21 Thymine DNA glycosylase (TDG) belongs to the UDG superfamily of DNA repair enzymes (Neddermann et al., 1996), but TDG also interacts with a number of transcriptional activators and coactivators as CBP/p300 (Tini et al., 2002) indicating its role in transcriptional regulation. Moreover, TDG binds histone acetyltransferases and DNA methyltransferases, and has been associated with DNA demethylation in gene promoters after being actively

26 transcribed, implicating a role in gene regulation. TDG is localized in the nucleus and in 27 some cells aggregated in the nucleoli, where it co-localized with Rep. All three proteins 
1 discussed in this latter paragraph bind to the Rep-proteins of both PCV, while c-myc and

2 syncoilin have been described to bind only to Rep of PCV2.

$4 \quad$ Proteins interacting with Cap

5 The capsid proteins of PCV1 and PCV2 differ only marginally in length, but in contrast to $6 \operatorname{Rep}(\mathrm{PCV} 1)$ and Rep(PCV2), they present a marked degree in amino acid sequence 7 diversion. Thus, they were candidates for pinning down a molecular marker indicative for the 8 differential pathogenicity of PCV1 and PCV2. Six cellular proteins (MKRN1, gC1qR, Par-4, 9 NAP1, NPM1 and Hsp40) were found with the yeast system to bind Cap of PCV1 as well as Cap(PCV2) (Finsterbusch et al., 2009).

Makorin ring finger protein 1 (MKRN1) is constitutively expressed in most human tissues. It contains several zinc finger motifs and is a member of the E3 ubiquitin ligase family. It has been reported to modulate telomere length homeostasis by ubiquitination and proteasomemediated degradation of the telomerase subunit hTERT (Kim et al., 2005). In the context of a viral infection, a recent study demonstrated that MKRN1 induces degradation of the capsid protein of West Nile virus (WNV) through ubiquitinylation and thereby prevents WNV-induced cell death via mitochondrial dysfunction and activation of caspase pathways (Ko et al., 2010). We observed reduced levels of Cap expression after co-expression of Cap and MKRN1, leading to the hypothesis that MKRN1 may promote the degradation of Cap. MKRN1 resides in dot-like structures in the nucleus. Upon co-expression with Cap, the fluorescence signal seemed more loosely distributed.

A very similar distribution effect was observed after co-expression of Cap and Hsp40, one of several cellular heat shock proteins (Hsp) induced to prevent cellular damage upon heat shock, UV irradiation and microbial or viral infection. Hsps are involved in apoptosis and immune response, they function as a chaperone thereby preventing protein aggregation and misfolding (Hartl, 1996). Hsp40 affects the replication of several viruses, it suppresses

27 hepatitis B virus replication (Sohn et al., 2006) and flavivirus replication (Yi et al., 2011) and 28 enhances replication of human papillomavirus (Lin et al., 2002) and human 
1 immunodeficiency virus HIV (Kumar and Mitra, 2005). It interacts with HIV-1 negative

2 regulatory factor (Nef) and is required for Nef-mediated increase in viral gene expression and

3 replication (Rawat and Mitra, 2011). For influenza virus, it has been reported that the

4 interferon-induced protein kinase (PKR) recruits p58IPK, a type III Hsp40 variant, which

5 inhibits PKR activity (Goodman et al., 2007). In contrast, the M2 protein of influenza virus

6 interact with Hsp40 variant Hdj1 to support PKR activation (Guan et al., 2010). Thus, the life

7 cycle of influenza virus involves manipulation of the antiviral response through the binding

8 and recruitment of two different types of Hsp40s with juxtaposed functions (Knox et al., 9 2011).

Another interesting protein that interacted with Cap of $\mathrm{PCV}$ is $\mathrm{gC1qR}$, an immune-receptor of the globular head of $\mathrm{C} 1 \mathrm{q} . \mathrm{C} 1 \mathrm{q}$ is the first component of the $\mathrm{C} 1$ complex in the complement system, which plays a significant role in innate immunity defense against microbes circulating in the blood of the infected host. Docking of $\mathrm{C} 1 \mathrm{q}$ to $\mathrm{gC1qR}$ leads to cellular defense activities. Hepatitis $\mathrm{C}$ virus (HCV) core protein differentially regulates $\mathrm{T}$ and $\mathrm{B}$ lymphocytes and inhibits monocyte IL-12 production through interaction with a complement receptor (gC1qR) expressed on these immune cells (Zhang et al., 2011). Receptor gC1qR also binds to HIV-1 Tat and Rev (Berro et al., 2006), the rubella capsid protein (Beatch et al., 2005) and the core proteins of adenovirus (Matthews and Russell, 1998). Binding promotes viral transcription, replication, capsid assembly and protein transport and prevents replication of white spot syndrome virus replication in crayfish (Watthanasurorot et al., 2010), serves as the receptor for Hantaan virus (Choi et al., 2008) and is known to modulate innate immunity by inhibiting RIG-I and MDA5-dependent antiviral signaling (Xu et al., 2009). The finding that the ligand of $\mathrm{gC} 1 \mathrm{qR}$ complement factor $\mathrm{C} 1 \mathrm{qB}$ interacts with the Cap-protein (Timmusk et al., 2006) led to the speculation, whether a ternary complex between Cap, C1qB and gC1qR may exist. When gC1qR was expressed with Rep, a most dramatic concentration of both proteins in a granular ring around the nucleoli was the consequence. 
1 protein WT1 (Johnstone et al., 1996). Recently, it was described that LMP-1 of Epstein-Barr

2 virus (EBV) represses Par-4 protein synthesis via the LMP-1/PI3 K/Akt signaling pathway

3 (Lee et al., 2009). Moreover, Par-4 targets the zipper interacting protein kinase to the actin

4 cytoskeleton of smooth muscle cells (Vetterkind and Morgan, 2008). Thus, the association of

5 Cap and Par-4 might play a role for transport or modification of viral proteins or particles. The

6 striate fluorescence signal of Par-4 in the cytoplasm was alleviated with co-expression of

7 Cap and Par-4 was partially re-distributed to the nucleus.

8 The Cap interacting proteins nucleosome assembly protein (NAP1) and nucleophosmin 9 (NPM1) are also involved in intracellular transport processes, both serve as chaperones and bind to basic proteins as histones. NPM1 is a multifunctional phosphoprotein, localized in the nucleoli. NPM1 has the ability to shuttle between the nucleus and cytoplasm. It is involved in 12 ribosome biogenesis (Savkur and Olson, 1998), and has been described as a histone chaperone implicated in chromatin organization and transcription control (Szebeni and Olson, 1999). It is also the most frequently mutated gene in acute myeloid leukemia (AML) (Falini et al., 2007; Sarek et al., 2010). NPM1 phosphorylation is involved in latency control of Karposi's sarcoma herpesvirus (KSHV): In KSHV-infected cells, NPM is phosphorylated by KSHV v-cyclin and the cellular CDK6 kinase. This modification modulates the interaction between NPM1 and the viral latency-associated nuclear antigen LANA, a repressor of lytic replication. Depletion of NPM1 leads to viral reactivation, and production of new infectious virus particles (Sarek et al., 2010). NPM1 showed increased levels after HPV infection, which was due to stabilization of NPM1 by the kinase AKT. Knockdown of NPM1 led to a decrease in cellular proliferation and an increase in the levels of the differentiation in infected cells, indicating a role for NPM in suppression of differentiation (McCloskey et al., 2010). In contrast, cells infected with Enterovirus 71 and Coxsackievirus A16 (CA16) the main etiological agents of hand, foot and mouth disease displayed a significant downregulation of

26 NPM1 (Lee et al., 2011), indicating that a common pathway utilisation of NPM in viral 27 infections cannot be discerned. Cap was seen more pronounced in the nucleoli after coexpression with NPM1. 
1 NAP1 is a histone chaperone which binds to all four core histones. Histone chaperones play

2 a prominent role in maintaining the critical balance between chromatin assembly and

3 disassembly and NAP1 has been implicated in $\mathrm{H} 2 \mathrm{~A} / \mathrm{H} 2 \mathrm{~B}$ dimer removal as a mechanism to

4 regulate DNA accessibility for subsequent transcription. NAP1 has also been shown to bind

5 CBP/p300 (Sharma and Nyborg, 2008). EBV nuclear antigen 1 interacts with NAP1, this

6 interaction affects in its functions in DNA replication and transcriptional activation (Wang and

7 Frappier 2009). Similar studies from HTLV-1 have shown that NAP1 mediates disassembly

8 of the acetylated nucleosomes from the promoter region of HTLV-1 (Nyborg et al., 2010;

9 Wang and Frappier, 2009). After expression of Cap, a proportion of NAP1 was redistributed to the cytoplasm, while the predominant signal was retained in the nucleus.

Cellular proteins binding to gpORF3:

13 Remarkably, gpORF3 is the only protein that differs in size and sequence between the two PCV variants: a longer protein of 207 aa is expressed from PCV1, while a 105 aa variant is seen in PCV2-infected cells. This renders gpORF3 an interesting candidate for the induction of pathogenesis. GpORF3 was reported to induce apoptosis and when PCV2(ORF3null) viruses were used to infect pigs, the animals had a delayed seroconversion and lower serum viral load (Liu et al., 2006), but a significant difference in the histological or gross lesions or the amount of PCV2-specific antigen in tissues was not observed in comparison to the wtPCV2. The properties of PCV1 and PCV2 gpORF3 were characterized with regard to induction of apoptosis. GpORF3(PCV1) induced more apoptotic cell death and was more toxic than gpORF3(PCV2) was. An experiment truncated ORF3 of PCV1 by insertion of a stop-codon and mutagenized the stop-codon present in ORF3 of PCV2, respectively, to adjust the different length of ORF3. The outcome revealed that the first 104 amino acids contain a domain capable of inducing cell death, whereas the $C$ terminus of gpORF3(PCV1) displays a domain possibly responsible for enhancing cell death (Chaiyakul et al., 2010). When Fenaux et al. produced chimeric viruses from PCV1 and PCV2 by inserting the cap gene of PCV1 into the PCV2 backbone, PCV2(cap PCV1), and vice versa PCV1(cap PCV2) 
1 by replacing the capsid gene of nonpathogenic PCV1 with that of PCV2 (Fenaux et al.,

2 2004). After inoculation of pigs with these constructs, seroconversion but no pathogenic

3 effects were observed with either of the chimeric viruses. Since gpORF3(PCV2) is comprised

4 in the construct PCV2(cap PCV1), these results suggest that the pathogenicity of PCV2 for

5 pigs is either not determined or not solely determined by the gpORF3.

6 The gpORF3 of PCV2 interacts with another ubiquitin E3 ligase, pPirh2 (porcine p53-induced

7 RING-H2), and competes with it for binding of p53 (Liu et al., 2007). Interaction induces the

8 alteration of the subcellular localization of pPirh2 and its destabilization. The subsequent

9 increase in p53 expression has been correlated with the induction of apoptosis

10 (Karuppannan et al., 2010).

11 Another porcine protein that binds to gpORF3 is a regulator of $G$ protein signalling (RGS) related to human RGS16 (huRGS16) (Timmusk et al., 2009). Transcription of poRGS16 was induced by a number of cell activators including mitogens, norepinephrine and interferon inducers. Other gpORF3 from PCV2 and from PCV1 were investigated: PCV2 variants bound to RGS16, while no binding was observed for PCV1. PCV2 gpORF3 co-localized with poRGS16 in LPS-activated porcine PBMC and poRGS16 may be assisting the translocation of gpORF3 into the nucleus.

\section{Host-virus interaction by protein - DNA interaction}

The origin of replication of PCV genome is located in the intergenic region between the cap and rep-genes (Mankertz and Hillenbrand, 2002) and contains an interferon-stimulated response element (ISRE)-like sequence. Using a yeast based ony hybrid assay, cellular proteins binding to this part of the genome were identified: Interferon-regulating Factor 1 (IRF-1a) and zinc finger protein ZNF683 bind to ISRE-Motiv of PCV1 and PCV2 (K. Doberstein, pers. communication), while transcription factor JunD binds to the Cre-Motif of PCV2. The implication of these unpublished observations was later on demonstrated, when Ramamoorthy et al. reported that ISRE mutation reduced viral replication in vitro and in vivo 
1 and elicited low antibody responses in PCV2 infection (Ramamoorthy et al., 2009;

2 Ramamoorthy et al., 2011).

Effects exerted by DNA or genome of PCV2

Another level of interaction between host and invading pathogens involves interference with the innate immune response of the host. This is often mediated by rather unspecific patterns of infectious agents, which are sensed as a danger signal by toll-like receptors. Some of these TLRs are activated by membrane constituents like LPS, others by nucleic acids with an unusual structure like dsRNA or CpG sequence motifs. The latter are present in bacterial and viral genomes and activate innate immunity via TLR9 signalling resulting in synthesis of interferons and other anti-inflammatory cytokines. The DNA genome of PCV2 contains several immuno-modulating motifs (Wikstrom et al., 2007), one of them was able to modulate porcine peripheral blood mononuclear cells in vitro. This sequence inhibited interferon- $\alpha$ production induced by immunostimulatory DNA. The effect was dependent on double strand and hairpin formation and the length of the palindrome but surprisingly not on the presence of a CpG motif.

Another effect exerted by PCV2 DNA was observed in plasmacytoid dendritic cells, where circular and dsDNA, the replicative form of the PCV2 genome, inhibited CpG activation via TLR9 but not via TLR7 (Balmelli et al., 2011). Interestingly, this effect did not occur, when PCV1 dsDNA was used instead of PCV2. The inhibition of cytokine synthesis in PDC and

21 MoDC treated with CpG-ODN was accompanied by a lack of actin polymerization and microfilament reorganisation, implying once that cytoskeletal reorganization was impaired in PCV2-infected cells. A decrease was also observed for endocytosis of PCV2 VLP, a process relying on microfilament formation. 


\section{$1 \quad$ Effects exerted by PCV2 on intracellular signaling cascades}

2 The inoculation of splenic macrophages (SM) with PCV2/PRRSV induced the de novo

3 expression and production of FasL in SMs but also concurrently increased the surface

4 expression of Fas and Fas/FasL-mediated apoptosis in co-cultured lymphocytes. The

5 interaction between Fas and FasL may contribute to the lymphoid depletion seen in PCV2

6 and PRRSV co-infected PMWS-affected pigs cells (Chang et al., 2007). The pathway with

7 which the upregulation of Fas and FasL is promoted has not yet been unravelled.

8 A very interesting study describes the effect of PCV2 on the NfkB pathway (Wei et al., 2008).

9 Upon PCV2 infection of PK15-cells, NfkB was activated. Infection resulted in degradation of 10 the NFBK inhibitor IkB and the subsequent translocation of NFkB into the nucleus. The

11 inhibition of the NfkB signalling pathway resulted in decreased expression of viral proteins and a reduced titre in the supernatant, indicative of an impaired viral replication. It will be interesting to learn about the trigger and the mechanism of NfkB induction by PCV2 and to compare it to PCV1.

Platform based approaches to identify differential regulation of the transcriptome and the proteome

Cellular protein response in PCV2 infected PK15 cells (Zhang et al., 2009) was studied by 2-

DE and MALDI/TOF. 34 proteins were found to be differentially expressed. Prominent alterations were found in proteins involved in organization of the cytoskeleton (ACT18, ACTG1, ACTB, TUBA1A, KRT8), stress response (HSP27), macromolecular biosynthesis (eEF2, ARPP0, RPA2, YARS2), RNA biosynthesis and processing (PNP, GMPS, HGPRT, HINT5), metabolic proteins (TALDO, PDHB, GALM, PPID, IDH3A, ENO1, 6PGL, PAGM, PAPSS1, PPP2R2A, ADI, PRDX4) and the ubiquitin-proteasome pathway (MOV40, PSMB3, PSME1, SGT1), signal transduction (NUP43, RAP2C, NCK1), and gene regulation (PHB). A different approach employing a combination of one and two dimensional gel electrophoresis in combination with ${ }^{16} \mathrm{O} /{ }^{18} \mathrm{O}$ labeling and mass spectrometry was used when colostrum deprived and Caesarian derived (CDCD) piglets were infected with PCV2 
1 (Ramirez-Boo et al., 2011). The proteomic study was performed on ingenual lymph nodes.

2 This approach led to the identification protein expression changes in 50 to 75 proteins

3 depending on the method. Most interestingly were several proteins, which were picked up by

4 both methods (ALB, HPX, CORO1A, HSP4A, SFRS1, FTL). NPM1, an interaction partner of

5 Cap was detected in this study as being upregulated. The proteins identified as differentially

6 expressed are involved in different processes as molecular transport, cell cycle regulation,

7 signal transduction, iron homeostasis, cell proliferation, and apoptosis. The two pathways

8 mostly affected were acute phase response (APR) signaling, NRF2-mediated oxidative

9 stress response and caveolar mediated endocytosis.

Differential Display-RT-PCR (DDRT-PCR) was used to identify differentially transcribed genes (Bratanich and Blanchetot, 2006). Several porcine genes were found to be upregulated in lymph node tissue but also in PK15 cells: (i) a gene similar to the hyaluronanmediated motility receptor (RHAMM), RHAMM is a microtubule-associated protein that maintains mitotic spindle pole integrity. It induces the Ras- and ERK-signalling pathways after binding of its ligand hyaluron. RHAMM is involved in elevation in solid and blood tumors and aggressive inflammatory response in rheumatoid arthritis (Jiang et al., 2010); (ii) a RNA splicing factor (SPF30) essential for assembly of the ribonucleoproteins into the spliceosome and putatively involved in the exosome pathway of heterochromatin silencing. SPF30 binds centromeric transcripts and locates at the centromeres in an RNA-dependent manner (Bernard et al., 2010); (iii) a RNA helicase comprising a DEAD box; (iv) PACSIN2 (Syndapin 2) belonging to the Src-homology $3(\mathrm{SH} 3)$-domain-containing proteins. These proteins function in the morphogenesis of neurons and interact with proteins implicated in vesicle trafficking and are involved in the regulation of tubulin polymerisation, endocytosis and actin dynamics (Hansen et al., 2011); (v) and a human kynurenine 3-monooxygenase enzyme, a mitochondrial enzyme in the tryptophan degradation pathway that has been linked directly to the pathophysiology of Huntington disease in humans (Giorgini et al., 2005). In a similar study performed in our group, we have found several genes up- and downregulated after 
1 infection with PCV1 and PCV2 (C. Adlhoch, pers. communication). Most of them as IL18 and

2 MHC-I are involved in immune response, STIP3 in signal transfer, and EHD3 und MyosinVb 3 in intracellular vesicle transport.

4 


\section{Conclusion:}

2 After several years of research addressing the interaction of PCV1 and PCV2 with their host, 3 many questions concerning these two viruses are not yet answered. Taken together all 4 results describing the interaction of PCV1 and PCV2 with the host cells at this moment do not point out a putative mechanism through which PCV2 exerts a pathogenic effect. The here described findings do not highlight a certain molecule, a pathway triggering PMWS and other PCVD. On the bright site, there are indications that certain cellular processes or machineries as cytoskeleton maintenance, intracellular signaling, RNA processing and endocytotic pathways are involved (Figure 1) and these aspects deserve our increased attention in the future. Although we have caught a first glimpse on the effects that are exerted by virusencoded proteins and the genome, we do not know yet how the cell reacts upon infection, how the virus adapts and evades the immune response. One would like to obtain more information about the pathogenic processes that lead to the onset of the disease. Therefore, further investigation should address the question which of the relevant connections between the cell and the PCV induce pathogenesis and which are employed to support the viral lifecycle but do not harm the organism. The multifunctional nature of PMWS and other PCVD does not render this task easier, but hopefully the studies summarized in this article will serve as a basis to continue the research leading to a better knowledge of the molecular pathogenesis of PCV1 and PCV2.

Acknowledgements: AM wishes to express her gratitude to the Robert Koch-Institute in person of Prof. Reinhard Burger and Prof. Martin Mielke and to all present and past members of her group for their continued commitment and support. 
Adams, D.J., van der Weyden, L., Mayeda, A., Stamm, S., Morris, B.J. and Rasko, J.E. (2001) ZNF265--a novel spliceosomal protein able to induce alternative splicing. J Cell Biol. 154(1), 25-32.

Allan, G., Meehan, B., Todd, D., Kennedy, S., McNeilly, F., Ellis, J., Clark, E.G., Harding, J., Espuna, E., Botner, A. and Charreyre, C. (1998) Novel porcine circoviruses from pigs with wasting disease syndromes. Vet Rec 142(17), 467-8.

Allday, M.J. (2009) How does Epstein-Barr virus (EBV) complement the activation of Myc in the pathogenesis of Burkitt's lymphoma? Semin Cancer Biol 19(6), 366-76.

Balmelli, C., Steiner, E., Moulin, H., Peduto, N., Herrmann, B., Summerfield, A. and McCullough, K. (2011) Porcine circovirus type 2 DNA influences cytoskeleton rearrangements in plasmacytoid and monocyte-derived dendritic cells. Immunology 132(1), 57-65.

Baylis, S.A., Finsterbusch, T., Bannert, N., Blumel, J. and Mankertz, A. (2011) Analysis of porcine circovirus type 1 detected in Rotarix vaccine. Vaccine 29(4), 690-7.

Beatch, M.D., Everitt, J.C., Law, L.J. and Hobman, T.C. (2005) Interactions between rubella virus capsid and host protein p32 are important for virus replication. J Virol. 79(16), 10807-20.

Bernard, P., Drogat, J., Dheur, S., Genier, S. and Javerzat, J.P. (2010) Splicing factor Spf30 assists exosome-mediated gene silencing in fission yeast. Mol Cell Biol 30(5), 1145 57.

Berro, R., Kehn, K., de la Fuente, C., Pumfery, A., Adair, R., Wade, J., Colberg-Poley, A.M., Hiscott, J. and Kashanchi, F. (2006) Acetylated Tat regulates human immunodeficiency virus type 1 splicing through its interaction with the splicing regulator p32. J Virol. 80(7), 3189-204.

Bratanich, A. and Blanchetot, A. (2006) A gene similar to the human hyaluronan-mediated motility receptor (RHAMM) gene is upregulated during Porcine Circovirus type 2 infection. Virus Genes 32(2), 145-52.

Chaiyakul, M., Hsu, K., Dardari, R., Marshall, F. and Czub, M. (2010) Cytotoxicity of ORF3 proteins from a nonpathogenic and a pathogenic porcine circovirus. J Virol 84(21), 11440-7.

Chang, H.W., Jeng, C.R., Lin, C.M., Liu, J.J., Chang, C.C., Tsai, Y.C., Chia, M.Y. and Pang, V.F. (2007) The involvement of Fas/FasL interaction in porcine circovirus type 2 and porcine reproductive and respiratory syndrome virus co-inoculation-associated lymphocyte apoptosis in vitro. Vet Microbiol 122(1-2), 72-82.

Choi, Y., Kwon, Y.C., Kim, S.I., Park, J.M., Lee, K.H. and Ahn, B.Y. (2008) A hantavirus causing hemorrhagic fever with renal syndrome requires $\mathrm{gC} 1 \mathrm{qR} / \mathrm{p} 32$ for efficient cell binding and infection. Virology 381(2), 178-83.

Clarke, W.T., Edwards, B., McCullagh, K.J., Kemp, M.W., Moorwood, C., Sherman, D.L., Burgess, M. and Davies, K.E. (2010) Syncoilin modulates peripherin filament networks and is necessary for large-calibre motor neurons. J Cell Sci 123(Pt 15), 2543-52.

Ellis, J., Hassard, L., Clark, E., Harding, J., Allan, G., Willson, P., Strokappe, J., Martin, K., McNeilly, F., Meehan, B., Todd, D. and Haines, D. (1998) Isolation of circovirus from lesions of pigs with postweaning multisystemic wasting syndrome. Can Vet J 39(1), 44-51.

Falini, B., Nicoletti, I., Bolli, N., Martelli, M.P., Liso, A., Gorello, P., Mandelli, F., Mecucci, C. and Martelli, M.F. (2007) Translocations and mutations involving the nucleophosmin (NPM1) gene in lymphomas and leukemias. Haematologica 92(4), 519-32. 
Fan, C., Ouyang, P., Timur, A.A., He, P., You, S.A., Hu, Y., Ke, T., Driscoll, D.J., Chen, Q. and Wang, Q.K. (2009) Novel roles of GATA1 in regulation of angiogenic factor AGGF1 and endothelial cell function. J Biol Chem 284(35), 23331-43.

Fenaux, M., Opriessnig, T., Halbur, P.G., Elvinger, F. and Meng, X.J. (2004) A chimeric porcine circovirus (PCV) with the immunogenic capsid gene of the pathogenic PCV type 2 (PCV2) cloned into the genomic backbone of the nonpathogenic PCV1 induces protective immunity against PCV2 infection in pigs. J Virol 78(12), 6297-303.

Finsterbusch, T. and Mankertz, A. (2009) Porcine circoviruses--small but powerful. Virus Res 143(2), 177-83.

Finsterbusch, T., Steinfeldt, T., Caliskan, R. and Mankertz, A. (2005) Analysis of the subcellular localization of the proteins Rep, Rep' and Cap of porcine circovirus type 1. Virology 343(1), 36-46.

Finsterbusch, T., Steinfeldt, T., Doberstein, K., Rodner, C. and Mankertz, A. (2009) Interaction of the replication proteins and the capsid protein of porcine circovirus type 1 and 2 with host proteins. Virology.

Giorgini, F., Guidetti, P., Nguyen, Q., Bennett, S.C. and Muchowski, P.J. (2005) A genomic screen in yeast implicates kynurenine 3-monooxygenase as a therapeutic target for Huntington disease. Nat Genet 37(5), 526-31.

Goodman, A.G., Smith, J.A., Balachandran, S., Perwitasari, O., Proll, S.C., Thomas, M.J., Korth, M.J., Barber, G.N., Schiff, L.A. and Katze, M.G. (2007) The cellular protein P58IPK regulates influenza virus mRNA translation and replication through a PKRmediated mechanism. J Virol 81(5), 2221-30.

Guan, Z., Liu, D., Mi, S., Zhang, J., Ye, Q., Wang, M., Gao, G.F. and Yan, J. (2010) Interaction of Hsp40 with influenza virus M2 protein: implications for PKR signaling pathway. Protein Cell 1(10), 944-55.

Hansen, C.G., Howard, G. and Nichols, B.J. (2011) Pacsin 2 is recruited to caveolae and functions in caveolar biogenesis. J Cell Sci 124(Pt 16), 2777-85.

Hartl, F.U. (1996) Molecular chaperones in cellular protein folding. Nature. 381(6583), 571-9.

Huang, X., Yuan, W., Huang, W., Bai, Y., Deng, Y., Zhu, C., Liang, P., Li, Y., Du, X., Liu, M., Wang, Y. and Wu, X. (2006) ZNF569, a novel KRAB-containing zinc finger protein, suppresses MAPK signaling pathway. Biochem Biophys Res Commun 346(3), 621-8.

Jiang, J., Casalegno-Garduno, R., Chen, H., Schmitt, A., Schmitt, M. and Maxwell, C.A. (2010) Multifunctional proteins bridge mitosis with motility and cancer with inflammation and arthritis. ScientificWorldJournal 10, 1244-57.

Johnstone, R.W., See, R.H., Sells, S.F., Wang, J., Muthukkumar, S., Englert, C., Haber, D.A., Licht, J.D., Sugrue, S.P., Roberts, T., Rangnekar, V.M. and Shi, Y. (1996) A novel repressor, par-4, modulates transcription and growth suppression functions of the Wilms' tumor suppressor WT1. Mol Cell Biol. 16(12), 6945-56.

Karuppannan, A.K., Liu, S., Jia, Q., Selvaraj, M. and Kwang, J. (2010) Porcine circovirus type 2 ORF3 protein competes with p53 in binding to Pirh2 and mediates the deregulation of p53 homeostasis. Virology 398(1), 1-11.

Kim, J.H., Park, S.M., Kang, M.R., Oh, S.Y., Lee, T.H., Muller, M.T. and Chung, I.K. (2005) Ubiquitin ligase MKRN1 modulates telomere length homeostasis through a proteolysis of hTERT. Genes Dev. 19(7), 776-81.

Knox, C., Luke, G.A., Blatch, G.L. and Pesce, E.R. (2011) Heat shock protein 40 (Hsp40) plays a key role in the virus life cycle. Virus Res.

Ko, A., Lee, E.W., Yeh, J.Y., Yang, M.R., Oh, W., Moon, J.S. and Song, J. (2010) MKRN1 induces degradation of West Nile virus capsid protein by functioning as an E3 ligase. $\mathrm{J}$ Virol 84(1), 426-36. 
Kumar, M. and Mitra, D. (2005) Heat shock protein 40 is necessary for human immunodeficiency virus-1 Nef-mediated enhancement of viral gene expression and replication. J Biol Chem. 280(48), 40041-50 Epub 2005 Sep 21.

Lee, J.J., Seah, J.B., Chow, V.T., Poh, C.L. and Tan, E.L. (2011) Comparative proteome analyses of host protein expression in response to Enterovirus 71 and Coxsackievirus A16 infections. J Proteomics.

Lee, J.W., Liu, P.F., Hsu, L.P., Chen, P.R., Chang, C.H. and Shih, W.L. (2009) EBV LMP-1 negatively regulates expression and pro-apoptotic activity of Par-4 in nasopharyngeal carcinoma cells. Cancer Lett 279(2), 193-201.

Li, R., Xi, Y., Liu, X., Chen, G., Wang, B., Jiang, L. and Li, W. (2009) Expression of IL1alpha, IL-6, TGF-beta, FasL and ZNF265 during sertoli cell infection by ureaplasma urealyticum. Cell Mol Immunol 6(3), 215-21.

Lin, B.Y., Makhov, A.M., Griffith, J.D., Broker, T.R. and Chow, L.T. (2002) Chaperone proteins abrogate inhibition of the human papillomavirus (HPV) E1 replicative helicase by the HPV E2 protein. Mol Cell Biol 22(18), 6592-604.

Liu, J., Chen, I., Du, Q., Chua, H. and Kwang, J. (2006) The ORF3 protein of porcine circovirus type 2 is involved in viral pathogenesis in vivo. J Virol 80(10), 5065-73.

Liu, J., Chen, I. and Kwang, J. (2005) Characterization of a previously unidentified viral protein in porcine circovirus type 2-infected cells and its role in virus-induced apoptosis. J Virol 79(13), 8262-74.

Liu, J., Zhu, Y., Chen, I., Lau, J., He, F., Lau, A., Wang, Z., Karuppannan, A.K. and Kwang, J. (2007) The ORF3 protein of porcine circovirus type 2 interacts with porcine ubiquitin E3 ligase Pirh2 and facilitates p53 expression in viral infection. J Virol 81(17), 9560-7.

Mankertz, A., Domingo, M., Folch, J.M., LeCann, P., Jestin, A., Segales, J., Chmielewicz, B., Plana-Duran, J. and Soike, D. (2000) Characterisation of PCV-2 isolates from Spain, Germany and France. Virus Res 66(1), 65-77.

Mankertz, A. and Hillenbrand, B. (2002) Analysis of transcription of Porcine circovirus type 1. J Gen Virol 83(Pt 11), 2743-51.

Mankertz, A., Mankertz, J., Wolf, K. and Buhk, H.J. (1998) Identification of a protein essential for replication of porcine circovirus. J Gen Virol 79 ( Pt 2), 381-4.

Matthews, D.A. and Russell, W.C. (1998) Adenovirus core protein V interacts with p32--a protein which is associated with both the mitochondria and the nucleus. J Gen Virol. 79(Pt 7), 1677-85.

McCloskey, R., Menges, C., Friedman, A., Patel, D. and McCance, D.J. (2010) Human papillomavirus type $16 \mathrm{E} 6 / \mathrm{E} 7$ upregulation of nucleophosmin is important for proliferation and inhibition of differentiation. J Virol 84(10), 5131-9.

Meehan, B.M., Creelan, J.L., McNulty, M.S. and Todd, D. (1997) Sequence of porcine circovirus DNA: affinities with plant circoviruses. J Gen Virol 78 ( Pt 1), 221-7.

Nawagitgul, P., Morozov, I., Bolin, S.R., Harms, P.A., Sorden, S.D. and Paul, P.S. (2000) Open reading frame 2 of porcine circovirus type 2 encodes a major capsid protein. $\mathbf{J}$ Gen Virol 81(Pt 9), 2281-7.

Neddermann, P., Gallinari, P., Lettieri, T., Schmid, D., Truong, O., Hsuan, J.J., Wiebauer, K. and Jiricny, J. (1996) Cloning and expression of human G/T mismatch-specific thymine-DNA glycosylase. J Biol Chem. 271(22), 12767-74.

Nyborg, J.K., Egan, D. and Sharma, N. (2010) The HTLV-1 Tax protein: revealing mechanisms of transcriptional activation through histone acetylation and nucleosome disassembly. Biochim Biophys Acta 1799(3-4), 266-74.

Oduber, C.E., van der Horst, C.M. and Hennekam, R.C. (2008) Klippel-Trenaunay syndrome: diagnostic criteria and hypothesis on etiology. Ann Plast Surg 60(2), 217-23. 
Ramamoorthy, S., Huang, F.F., Huang, Y.W. and Meng, X.J. (2009) Interferon-mediated enhancement of in vitro replication of porcine circovirus type 2 is influenced by an interferon-stimulated response element in the PCV2 genome. Virus Res 145(2), 23643.

Ramamoorthy, S., Opriessnig, T., Pal, N., Huang, F.F. and Meng, X.J. (2011) Effect of an interferon-stimulated response element (ISRE) mutant of porcine circovirus type 2 (PCV2) on PCV2-induced pathological lesions in a porcine reproductive and respiratory syndrome virus (PRRSV) co-infection model. Vet Microbiol 147(1-2), 4958.

Ramirez-Boo, M., Nunez, E., Jorge, I., Navarro, P., Fernandes, L.T., Segales, J., Garrido, J.J., Vazquez, J. and Moreno, A. (2011) Quantitative proteomics by 2-DE, (16) O/(18) O labelling and linear ion trap mass spectrometry analysis of lymph nodes from piglets inoculated by porcine circovirus type 2 . Proteomics.

Rawat, P. and Mitra, D. (2011) Cellular heat shock factor 1 positively regulates human immunodeficiency virus-1 gene expression and replication by two distinct pathways. Nucleic Acids Res 39(14), 5879-92.

Sarek, G., Jarviluoma, A., Moore, H.M., Tojkander, S., Vartia, S., Biberfeld, P., Laiho, M. and Ojala, P.M. (2010) Nucleophosmin phosphorylation by v-cyclin-CDK6 controls KSHV latency. PLoS Pathog 6(3), e1000818.

Savkur, R.S. and Olson, M.O. (1998) Preferential cleavage in pre-ribosomal RNA byprotein B23 endoribonuclease. Nucleic Acids Res. 26(19), 4508-15.

Sells, S.F., Wood, D.P., Jr., Joshi-Barve, S.S., Muthukumar, S., Jacob, R.J., Crist, S.A., Humphreys, S. and Rangnekar, V.M. (1994) Commonality of the gene programs induced by effectors of apoptosis in androgen-dependent and -independent prostate cells. Cell Growth Differ. 5(4), 457-66.

Sharma, N. and Nyborg, J.K. (2008) The coactivators CBP/p300 and the histone chaperone NAP1 promote transcription-independent nucleosome eviction at the HTLV-1 promoter. Proc Natl Acad Sci U S A 105(23), 7959-63.

Sohn, S.Y., Kim, S.B., Kim, J. and Ahn, B.Y. (2006) Negative regulation of hepatitis B virus replication by cellular Hsp40/DnaJ proteins through destabilization of viral core and $\mathrm{X}$ proteins. J Gen Virol. 87(Pt 7), 1883-91.

Steinfeldt, T., Finsterbusch, T. and Mankertz, A. (2006) Demonstration of nicking/joining activity at the origin of DNA replication associated with the rep and rep' proteins of porcine circovirus type 1. J Virol 80(13), 6225-34.

Szebeni, A. and Olson, M.O. (1999) Nucleolar protein B23 has molecular chaperone activities. Protein Sci. 8(4), 905-12.

Timmusk, S., Fossum, C. and Berg, M. (2006) Porcine circovirus type 2 replicase binds the capsid protein and an intermediate filament-like protein. J Gen Virol. 87(Pt 11), 321523.

Timmusk, S., Merlot, E., Lovgren, T., Jarvekulg, L., Berg, M. and Fossum, C. (2009) Regulator of $\mathrm{G}$ protein signalling 16 is a target for a porcine circovirus type 2 protein. J Gen Virol 90(Pt 10), 2425-36.

Tini, M., Benecke, A., Um, S.J., Torchia, J., Evans, R.M. and Chambon, P. (2002) Association of CBP/p300 acetylase and thymine DNA glycosylase links DNA repair and transcription. Mol Cell. 9(2), 265-77.

Tischer, I., Mields, W., Wolff, D., Vagt, M. and Griem, W. (1986) Studies on epidemiology and pathogenicity of porcine circovirus. Arch Virol 91(3-4), 271-6.

Vetterkind, S. and Morgan, K.G. (2008) The Pro-Apoptotic Protein Par-4 Facilitates Vascular Contractility by Cytoskeletal Targeting of Zipk. J Cell Mol Med 24, 24. 
Wakayama, Y., Matsuzaki, Y., Yamashita, S., Inoue, M., Jimi, T., Hara, H., Unaki, A., Iijima, S. and Masaki, H. (2010) Dysbindin, syncoilin, and beta-synemin mRNA levels in dystrophic muscles. Int J Neurosci 120(2), 144-9.

Wang, S. and Frappier, L. (2009) Nucleosome assembly proteins bind to Epstein-Barr virus nuclear antigen 1 and affect its functions in DNA replication and transcriptional activation. J Virol 83(22), 11704-14.

Watthanasurorot, A., Jiravanichpaisal, P., Soderhall, I. and Soderhall, K. (2010) A gC1qR prevents white spot syndrome virus replication in the freshwater crayfish Pacifastacus leniusculus. J Virol 84(20), 10844-51.

Wei, L., Kwang, J., Wang, J., Shi, L., Yang, B., Li, Y. and Liu, J. (2008) Porcine circovirus type 2 induces the activation of nuclear factor kappa B by IkappaBalpha degradation. Virology 378(1), 177-84.

Wikstrom, F.H., Meehan, B.M., Berg, M., Timmusk, S., Elving, J., Fuxler, L., Magnusson, M., Allan, G.M., McNeilly, F. and Fossum, C. (2007) Structure-dependent modulation of alpha interferon production by porcine circovirus 2 oligodeoxyribonucleotide and CpG DNAs in porcine peripheral blood mononuclear cells. J Virol 81(10), 4919-27.

Xu, L., Xiao, N., Liu, F., Ren, H. and Gu, J. (2009) Inhibition of RIG-I and MDA5-dependent antiviral response by gC1qR at mitochondria. Proc Natl Acad Sci U S A 106(5), 15305.

Yi, Z., Sperzel, L., Nurnberger, C., Bredenbeek, P.J., Lubick, K.J., Best, S.M., Stoyanov, C.T., Law, L.M., Yuan, Z., Rice, C.M. and MacDonald, M.R. (2011) Identification and characterization of the host protein DNAJC14 as a broadly active flavivirus replication modulator. PLoS Pathog 7(1), e1001255.

Zhang, X., Zhou, J., Wu, Y., Zheng, X., Ma, G., Wang, Z., Jin, Y., He, J. and Yan, Y. (2009) Differential proteome analysis of host cells infected with porcine circovirus type 2 . J Proteome Res 8(11), 5111-9.

Zhang, Y., Ma, C.J., Ni, L., Zhang, C.L., Wu, X.Y., Kumaraguru, U., Li, C.F., Moorman, J.P. and Yao, Z.Q. (2011) Cross-talk between programmed death-1 and suppressor of cytokine signaling-1 in inhibition of IL-12 production by monocytes/macrophages in hepatitis C virus infection. J Immunol 186(5), 3093-103. 
$-21-$

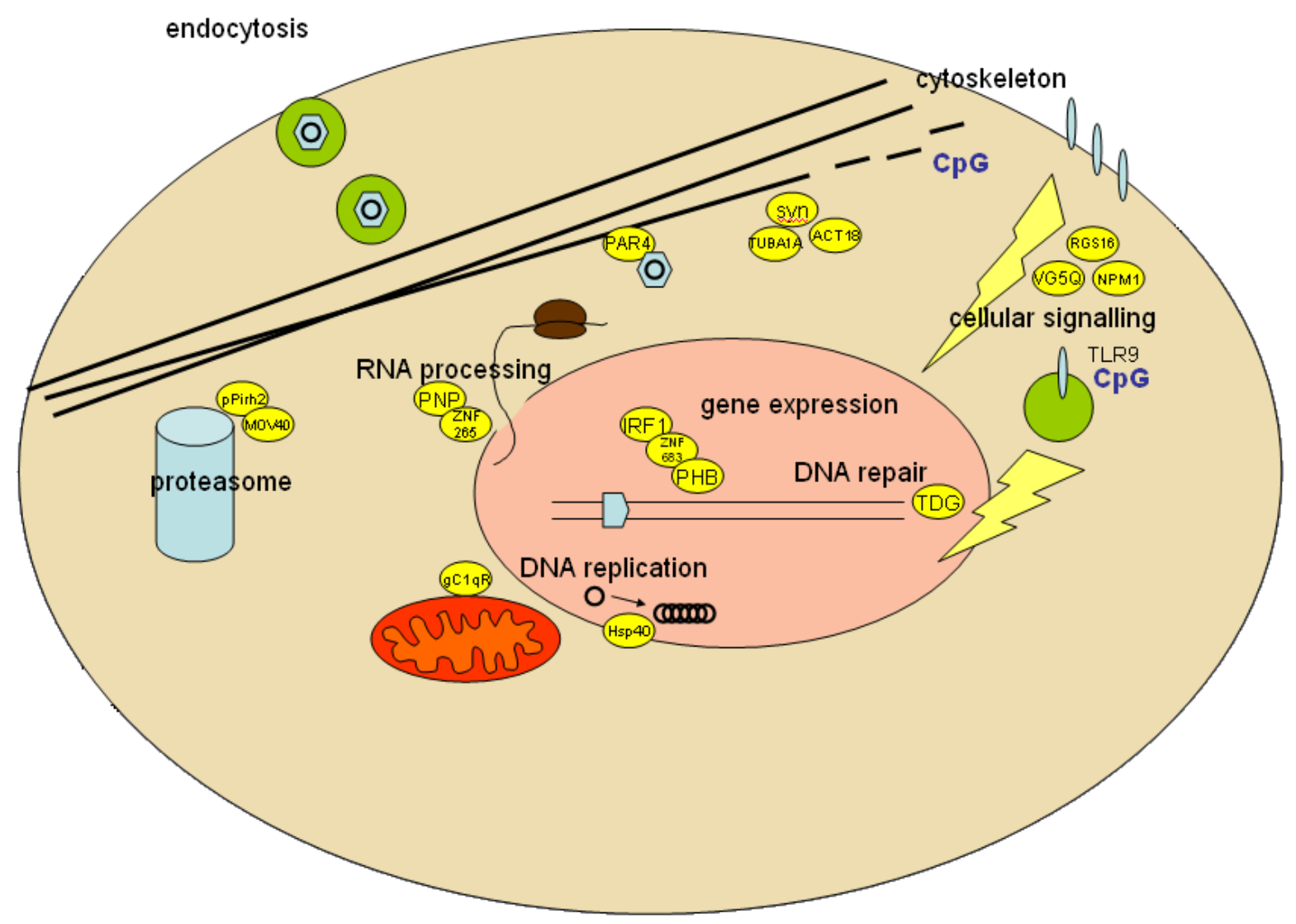

